\title{
GLI IDROCARBURI TRA IMPATTO ECONOMICO, RICERCA E PROSPETTIVE
}

\author{
ROBERTO ANIELLO CALABRÒ (*)
}

Nota presentata dal m.e. Giuseppe Cassinis

(Adunanza del 20 gennaio 2011)

\begin{abstract}
SuNTO. - Gli idrocarburi rappresentano l'unica fonte energetica non rinnovabile su cui si basa l'economia mondiale e che ne condiziona anche lo sviluppo. I principali paese produttori sono localizzati in medio-oriente per quanto riguarda il petrolio e la Russia ed ancora il medio oriente per il gas. La ricerca petrolifera è iniziata a metà del $1800 \mathrm{e}$ ha avuto uno sviluppo significativo durante la seconda guerra mondiale prima e durante lo sviluppo post bellico, legato alla ricostruzione. La formazione degli idrocarburi è legata alla presenza di rocce a grana fine (argilliti) della sostanza organica (1-14\%), di origine vegetale (alghe e foglie) ed animale (microorganismi). Quando le rocce che contengono questa sostanza organica sono soggette a carichi litostatici elevati e la temperatura raggiunge circa $90^{\circ}$, la sostanza organica comincia a trasformarsi ed a temperatura di circa $120^{\circ}$, comincia a formarsi prima il petrolio e ad una temperatura di circa $140^{\circ}$, si genera il gas. Dopo la generazione gli idrocarburi si accumulano in rocce porose (reservoir), caratterizzate da una geometria che ne permetta l'accumulo. Soprastante al reservoir è sempre presenta una roccia a grana fine ed impermeabile che non consente agli idrocarburi di disperdersi. Negli ultimi anni, si è cominciato ad investire anche sugli idrocarburi non convenzionali, rappresentati in particolare modo dalle gas shale e dal "oil sand". Ad oggi le riserve di idrocarburi accertate sono pari a 1333 di miliardi di barili di olio e 187.490 miliardi di metri cubi di gas, per una durata stimabile in circa 50 anni. Se agli idrocarburi convenzionali sommiamo quelli non convenzionali le riserve disponibili e se teniamo conto dei consumi attuali, possiamo stimare che avremo ancora idrocarburi per circa 100 anni.
\end{abstract}

(*) Edison S.p.A. Foro Bonaparte 31, 20121 Milano, Italy.

E-mail: roberto.calabro@edison.it 
ABSTRACT. - Fossil fuels are the only non-renewable energy sources on which the world economy is based directly affect development. The main producer countries are located in the Middle-East (oil) and Russia and Middle East (gas). The oil search began in 1860 and has had a significant development during the Second World War, and in particularly in the post-war development, linked to the reconstruction. The formation of hydrocarbons is linked to the presence of fine-grained rocks (shales and limestone) that contain organic matter (1-14\%), of vegetable (such as algae and leaves) and animal origin (microorganisms). When the rocks that contain this organic substance are buried subjected to high lithostatic load and the temperature reaches about $90^{\circ} \mathrm{C}$, the organic matter begins to be transformed and at a temperature of about $120^{\circ} \mathrm{C}$, begins to form oil, and at a temperature of about $140^{\circ} \mathrm{C}$, gas is generated. After generation and migration the hydrocarbons accumulate in porous rocks (reservoirs), characterized by a geometry structures allowing their accumulation. Above the reservoir there should be a fine-grained and impermeable rock, that does not allow oil to disperse. In recent years, oil \& gas companies have started to invest on non-conventional hydrocarbons, in particular, represented by shale gas and oil sands. To date, proven oil reserves amounted to 1.333 billion barrels of oil and 187.490 billion cubic meters of gas, reserves estimated to last for about approximately 50 years. If we add unconventional hydrocarbon reserves available and take into account the current consumption rate we could estimate that we would have oil for about 100 years more.

\section{INTRODUZIONE}

La civiltà industriale dipende in larga misura dai derivati del petrolio. Solo negli anni Settanta la riduzione delle forniture petrolifere costrinse i paesi industrializzati a cercare prodotti alternativi al petrolio, sia nel settore della produzione di energia, sia in quello industriale.

Malgrado le crisi petrolifere degli ultimi decenni, gli idrocarburi rappresentano tutt'ora la principale fonte energetica non rinnovabile e per la loro importanza condizionano pesantemente l'economia mondiale in ogni filiera produttiva.

Anche se le riserve sono ancora significative, la formazione degli Idrocarburi è un processo molto complesso e il loro accumulo è avvenuto sempre in particolare aree petrolifere come risultato della perfetta integrazione tra fenomeni fisici, chimici e geologici. Alla base di tutto vi è naturalmente la deposizione di sostanza organica, costituita da microorganismi e materiale vegetale, in ambienti marini o deltizi privi di ossigeno. La successiva compattazione associata all'espulsione dell'acqua, al progressivo seppellimento ed aumento di pressione e temperature $\left(120^{\circ} \mathrm{C}-150^{\circ} \mathrm{C}\right)$, porta la sostanza organica a trasformarsi ed a generare idrocarburi, in un processo chimico-fisico che può durare anche deci- 
ne di milioni di anni. Contemporaneamente alla generazione, gli idrocarburi tendono a migrare verso l'alto o lateralmente a causa della loro bassa densità. Se durante la migrazione trovano delle strutture dove possono accumularsi, danno origine a veri e propri giacimenti. Nel caso in cui invece raggiungano la superficie, si disperdono e non danno origine ad alcun accumulo.

\section{BREVI CENNI STORICI}

Dal punto di vista storico le prime testimonianze dell'uso del petrolio risalgono alla civiltà cinese (3000 anni fa) ma alcuni riferimenti si trovano anche nel Vecchio Testamento (Genesi), fino ad arrivare nel Medioevo, come descritto nel Milione di Marco Polo, dove sono riportate notizie in merito al commercio di idrocarburi nell'area dell'odierno Azerbaigian.

In tempi più recenti, le testimonianze dell'uso degli idrocarburi li abbiamo in Galizia nel XVI secolo, dove l'olio veniva usato come medicinale e per le lampade, in Birmania, dove nel secolo XIX si producevano circa 200.000 barili l'anno e nel Nord America, dove Samuel Kier (commerciante ed inventore canadese), commercializzava il petrolio sia come sostanza medicinale che come combustibile per lampade.

Anche se gli idrocarburi erano già conosciuti dall'uomo, la storia moderna della ricerca e del loro sfruttamento inizia ufficialmente con la metà dell'ottocento, quando il 27 Agosto del 1859 il colonnello Edwin Laurentine Drake, perforò il primo pozzo petrolifero a Titusville (Pennsylvania) (Fig. 1).

Il pozzo raggiunse una profondità di circa $25 \mathrm{~m}$, con una produzione giornaliera di circa venti barili di olio.

E sempre in questo periodo che il petrolio, per essere trasportato, veniva messo nei barili di whisky che avevano una capacità di circa 159 litri (42 galloni).

Bisogna aspettare la prima guerra mondiale, però, per registrare un utilizzo massiccio degli idrocarburi, fino a raggiungere un consumo di circa 15 milioni di barili nel 1920.

I principali Paesi produttori nei primi decenni del secolo scorso erano gli USA, il Canada, la Russia, il Messico, l'Australia, la Germania e la Romania. 


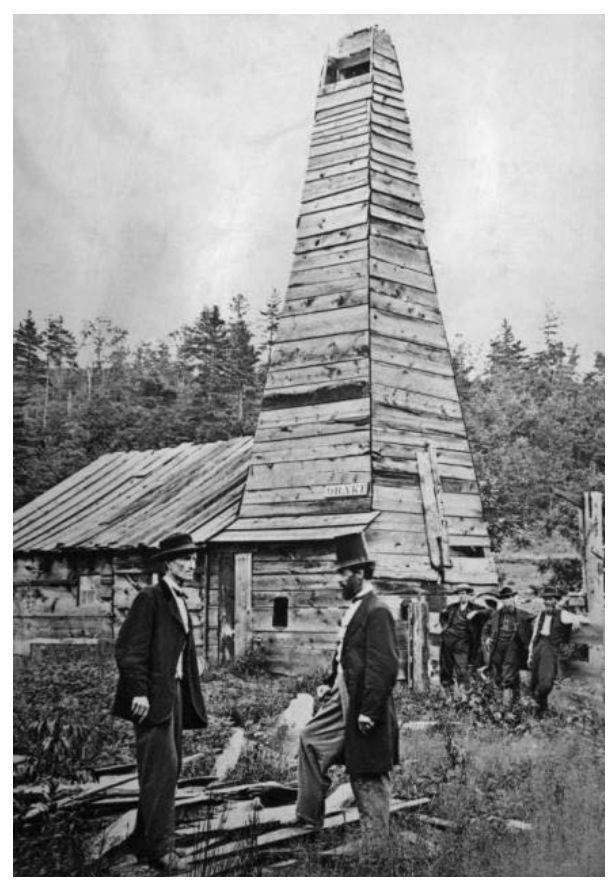

Fig. 1 - Foto del 1859 relativa al primo pozzo perforato dal Colonnello Edwin Laurentine Drake (a destra).

Negli anni ‘ 40 del secolo scorso, si registrò un aumento della produzione grazie ad un secondo gruppo di paesi, rappresentato da: Arabia Saudita, Iran, Iraq, Bahrein, Brunei, Ecuador e Venezuela.

Tra gli anni quaranta e settanta del novecento, entrarono sul mercato anche il Kuwait, il Qatar, la Nigeria, la Libia, l'Algeria, l'Angola, il Gabon e la Norvegia.

L'aumento significativo dei paesi produttori è strettamente legato all'utilizzo di metodologie di indagini sempre più sofisticate, come ad esempio la sismica a riflessione, la magnetotellurica, gli studi da satelliti, all'utilizzo di computer sempre più veloci e potenti, allo sviluppo di software dedicati, che hanno consentito la gestione di milioni di informazioni al secondo e allo sviluppo delle tecnologie legate alla perforazione, sfruttamento e trasporto degli idrocarburi.

Ciò ha permesso di allargare la ricerca anche alle aree marine (offshore), riuscendo a perforare pozzi a profondità del fondo marino di $3000 \mathrm{~m}$ e alle aree artiche. 


\section{GLI IDROCARBURI}

L'obiettivo della ricerca petrolifera è quello di valutare il potenziale petrolifero di una determinata area e quindi di riconoscere nel sottosuolo la presenza di eventuali trappole, dove possono essersi accumulati degli idrocarburi.

A tal proposito, i geologi del petrolio definiscono "Prospect" quelle trappole nelle quali la probabilità di fare una scoperta è particolarmente alta, mentre con il termine "Lead" definiscono le aree in cui potenzialmente potrebbero esserci degli idrocarburi, ma dove è necessario comunque un supplemento di indagine.

L'accumulo degli idrocarburi in una trappola è il risultato di una perfetta integrazione e della somma di alcune condizioni geologiche.

Più in particolare:

1. presenza di rocce ricche in sostanza organica con percentuale variabile dal 1 al $15 \%$, sottoposte a condizioni di temperatura e di pressioni tali da favorire la generazione di idrocarburi;

2. espulsione degli idrocarburi e loro migrazione;

3. presenza di rocce serbatoio (reservoir) caratterizzate da alta porosità e permeabilità dove gli idrocarburi possono accumularsi;

4. esistenza di coperture impermeabili che permettano all'olio accumulato di non disperdersi nelle rocce adiacenti;

5. integrità della roccia serbatoio e della copertura nel tempo, in modo che eventi tettonici deformativi successivi all'accumulo non causino la dispersione degli idrocarburi.

\section{LE ROCCE MADRI}

Le rocce madri hanno un ruolo fondamentale nella generazione degli idrocarburi in quanto contengono quella sostanza organica che sottoposta a determinate condizioni di temperatura e pressione può dare origine a gas e petrolio.

Le rocce madri (o source rock) sono rappresentate in genere da rocce silicoclastiche a grana fine (argilliti) o carbonatiche, caratterizzate da un alto contenuto in sostanza organica variabile dal 1 al $15 \%$, valore espresso dal TOC (Total Organic Carbon).

$\mathrm{La}$ deposizione di queste rocce avviene in un ambiente marino o lacustre (Fig. 2), caratterizzato da bassa energia e da scarso contenuto 
di ossigeno, in condizioni quindi in cui la materia organica può essere conservata (condizioni anaerobiche).

L'assoluta mancanza di ossigeno impedisce infatti i processi di ossidazione chimica e quindi la rapida decomposizione della materia organica.

In alcuni casi i batteri presenti negli ambienti anaerobi, durante la fase di attacco della sostanza organica, producono solfuro di idrogeno $\left(\mathrm{H}_{2} \mathrm{~S}\right)$ come per esempio nelle rocce madri carbonatiche, dove questo processo è molto più accentuato.

Gli oli che si ottengono da queste rocce madri sono spesso caratterizzati da un alto tenore in solfuri.
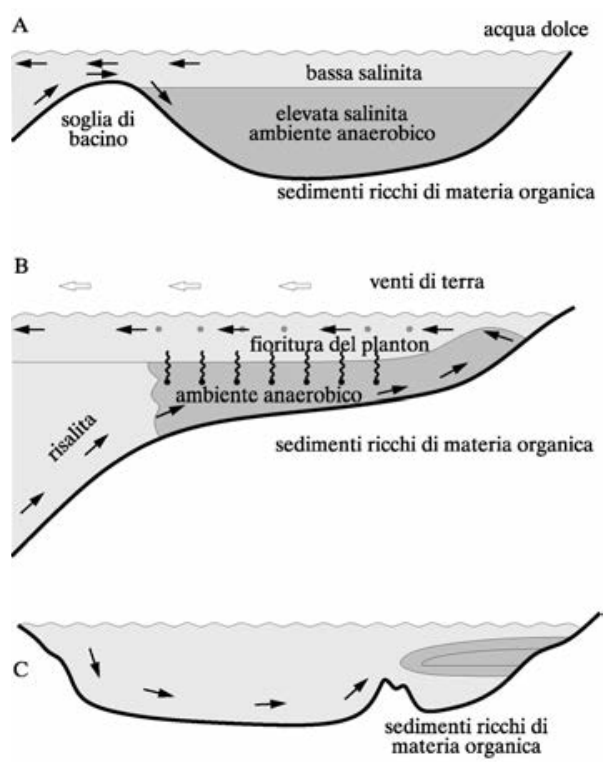

Fig. 2 - Schema semplificato dell'ambiente di sedimentazione della sostanza organica, in un ambiente a scarsa ossigenazione che darà origine alle source rock.

(Demaison G.J., Moore G.T., 1980, modificato).

\section{LA SOSTANZA ORGANICA}

La formazione degli idrocarburi è strettamente collegata alla disponibilità di atomi di carbonio, i quali devono essere "preservati" dall'azione dell'ossigeno in quanto ne causerebbe la trasformazione in ossidi di carbonio e altri composti organici. 
I principali produttori di carbonio organico possono essere sia organismi di origine animale e vegetale, quali il plancton e il fitoplancton (diatomee, dinoflagellati, nano plancton), sia parti cellulari delle alghe, lipidi, membrane, cuticole, spore e pollini.

Il contributo degli organismi più grandi, come i pesci e gli animali terrestri è trascurabile, mentre le piante ad alto fusto, che sono più resistenti alla degradazione atmosferica e quindi hanno più tempo a disposizione per trovare un ambiente capace di preservarle, in genere danno vita a depositi di carbone.

La trasformazione della sostanza organica, in kerogene e poi in idrocarburi avviene grazie al progressivo seppellimento e al contestuale aumento della temperatura e della pressione, processo che può durare da qualche decina a un centinaio di milioni di anni.

Negli ultimi decenni la geochimica ha permesso di caratterizzate meglio il kerogene (Tab. 1), attraverso una determinazione del rapporto del contenuto degli atomi di idrogeno $(\mathrm{H})$, carbonio $(\mathrm{C})$ e ossigeno (O). Sulla base di queste analisi è stato possibile distinguere 5 diversi tipi (Tab. 1):

- il kerogene di tipo I è costituito in gran parte da sostanza organica amorfa, cyanobatteri, alghe e parti di piante terrestri. Ha un rapporto $\mathrm{H} / \mathrm{C}>1,4$ e un rapporto $\mathrm{O} / \mathrm{C}<0,15$ L'ambiente di deposizione è tipicamente di tipo lacustre o transizionale (delta fluviali). Da questo kerogene si genera solitamente dell'olio;

- il kerogene di tipo II è costituito da sporinite (pollini e spore), cutinite (cuticole di piante terrestri), resine (piante terrestri da animali in decomposizione) e da leptinite (lipidi di piante terrestri e da alghe). Ha un rapporto $\mathrm{H} / \mathrm{C}<1,25$ e un rapporto $\mathrm{O} / \mathrm{C}$ compreso tra 0,03 e 0,18 . La tendenza di questo kerogene è quella di produrre olio e una parte significativa di gas;

- il kerogene II-S è uguale al precedente ma con un alto tenore in zolfo;

- il kerogene di tipo III è costituito da piante terrestri, cellulosa, carboidrati che costituiscono la parte rigida delle piante terrestri, lignina, terpeni e fenoli. Ha un rapporto $\mathrm{H} / \mathrm{C}<1$ e un rapporto $\mathrm{O} / \mathrm{C}$ compreso tra 0,03 e 0,3 . Generalmente questo kerogene produce carbone o gas;

- il kerogene di tipo IV contiene materia organica in parte decomposta nella forma di idrocarburi aromatici policiclici. Questo kerogene non produce mai idrocarburi. 
Tab. 1

\begin{tabular}{|c|c|c|c|c|c|c|}
\hline $\begin{array}{l}\text { Tipo di } \\
\text { Kerogene }\end{array}$ & $\begin{array}{l}\text { Indice di } \\
\text { idrogeno }\end{array}$ & $\begin{array}{l}\text { Indice di } \\
\text { ossigeno }\end{array}$ & $\begin{array}{c}\text { Rapporto } \\
\text { H/C }\end{array}$ & $\begin{array}{l}\text { Materia } \\
\text { organica }\end{array}$ & $\begin{array}{c}\text { Ambiente } \\
\text { deposizionale }\end{array}$ & $\begin{array}{l}\text { Prodotti } \\
\text { principali }\end{array}$ \\
\hline I & $700-1000$ & $10-40$ & 1,4 & $\begin{array}{c}\text { Algale } \\
\text { amorfa } \\
\text { raramente } \\
\text { terrestre }\end{array}$ & $\begin{array}{l}\text { Anossico } \\
\text { lacustre } \\
\text { raramente } \\
\text { marino }\end{array}$ & Olio \\
\hline II & $350-700$ & $20-60$ & $1,2-1,4$ & $\begin{array}{c}\text { Algale } \\
\text { amorfa } \\
\text { terrestre }\end{array}$ & $\begin{array}{l}\text { Anossico } \\
\text { marino }\end{array}$ & Olio \\
\hline II/III & $200-350$ & $40-80$ & $1-1,2$ & $\begin{array}{c}\text { Mista } \\
\text { marina } \\
\text { terrestre }\end{array}$ & $\begin{array}{c}\text { Transizionale } \\
\text { deltizio }\end{array}$ & Olio/Gas \\
\hline IV & $50-200$ & $50-150$ & $0,7-1$ & $\begin{array}{c}\text { Terrestre } \\
\text { vitrinite } \\
\text { algale alterata }\end{array}$ & $\begin{array}{l}\text { Piattaforma } \\
\text { scarpata }\end{array}$ & Gas \\
\hline $\mathrm{V}$ & $<50$ & $20-200$ & $1,4-0,7$ & $\begin{array}{l}\text { Altamente } \\
\text { ossidata } \\
\text { rimaneggiata }\end{array}$ & $\begin{array}{l}\text { Altamente } \\
\text { ossidata } \\
\text { ovunque }\end{array}$ & Gas secco \\
\hline
\end{tabular}

I processi che portano alla trasformazione della sostanza organica in kerogene e poi in idrocarburi sono quindi da mettere in relazione al gradiente di temperatura e di pressione. Più in particolare, a circa $1 \mathrm{~km}$ di profondità e ad una temperatura di almeno $50^{\circ} \mathrm{C}$, comincia la diagenesi, processo attraverso il quale i sedimenti tendono a litificarsi e a perdere l'acqua contenuta nei pori.

Con il progressivo seppellimento e quindi aumento di temperatura e pressione, si può arrivare ad un vero e proprio metamorfismo di basso grado $\left(6-7 \mathrm{~km}-\mathrm{T} 200^{\circ} \mathrm{C}\right)$.

Durante la diagenesi il sedimento e la materia organica, subiscono quindi una compattazione consentendo ai batteri presenti nel terreno di "fermentare" la sostanza organica, producendo anidride carbonica e idrocarburi.

Al termine della diagenesi la sostanza organica è in parte ossidata, in parte riciclata dai microrganismi, in parte è stata fermentata producendo metano ed infine parte si trasforma in kerogene.

A seguito di un ulteriore incremento della temperatura si arriva alla fase di catagenesi dove il kerogene passa allo stato amorfo in cui 
sono presenti macromolecole formate principalmente da carbonio ed idrogeno, con una piccola percentuale di ossigeno, zolfo e azoto.

Ad un successivo aumento della temperatura, il kerogene continua a trasformarsi eliminando le molecole più leggere e relativamente ricche di $\mathrm{O}$ e $\mathrm{H}$, assumendo una struttura più ordinata e stabile.

Nella fase finale della catagenesi il kerogene completa la sua maturazione a circa $120^{\circ} \mathrm{C} / 150^{\circ} \mathrm{C}$ e diversi $\mathrm{km}$ di profondità con pressioni che possono raggiungere le 1000 atmosfere.

A queste condizioni avviene il cracking, indotto dal solo aumento di temperatura.

Durante questa fase il kerogene subisce la rottura della macromolecola originale formando molecole di idrocarburo che, essendo molto meno dense della macromolecola di partenza, tenderanno a migrare verso l'alto e ad accumularsi laddove troveranno delle barriere rocciose impermeabili (trappole).

Il kerogene rimanente si arricchirà sempre più in carbonio, con un numero sempre minore di atomi suscettibili di rottura.

Il cracking inizialmente produce solo petrolio, nelle fasi conclusive, invece, genera gas e condensati (composti leggeri in forma gassosa nel sottosuolo e liquidi alle condizioni ambientali).

La produzione di petrolio avviene entro un determinato intervallo di temperatura: una minima di circa $60^{\circ} \mathrm{C}$ e ad una profondità di circa 2000-2500 m, in cui inizia la fase di diagenesi al cui termine si genera il kerogene, ed una massima tra i $120^{\circ} \mathrm{C}-150^{\circ} \mathrm{C}$ in cui il kerogene subisce il fenomeno di cracking.

Queste sono le due soglie termiche che delimitano la cosiddetta finestra dell'olio, ossia l'intervallo di profondità e temperatura calcolate con un gradiente geotermico medio in cui la roccia madre produce la massima quantità di petrolio.

Alla soglia del metamorfismo (circa 5-6 Km di profondità e temperatura di circa $200^{\circ} \mathrm{C}$ ) il kerogene diventa un residuo carbonioso grafitico. In queste condizioni non esiste alcuna possibilità di generare ulteriore petrolio.

\section{LA MIGRAZIONE}

La migrazione è uno dei processi più importanti che permette agli idrocarburi di muoversi dall'area di generazione (kitchen) a 
quello di accumulo (Fig. 3), ed avviene in seguito al progressivo aumento del carico litostatico che ha come effetto un aumento della pressione e quindi la compattazione delle rocce, con diminuzione della porosità.

In genere le principali rocce madri sono quelle argillose, che per loro composizione, contengono una grande quantità di acqua.

Con il progressivo seppellimento l'acqua e gli idrocarburi contenuti vengono espulsi (si stima che a $2000 \mathrm{~m}$ di profondità si possa espellere il $90 \%$ di fluidi, con una perdita di volume del $50 \%$ circa.

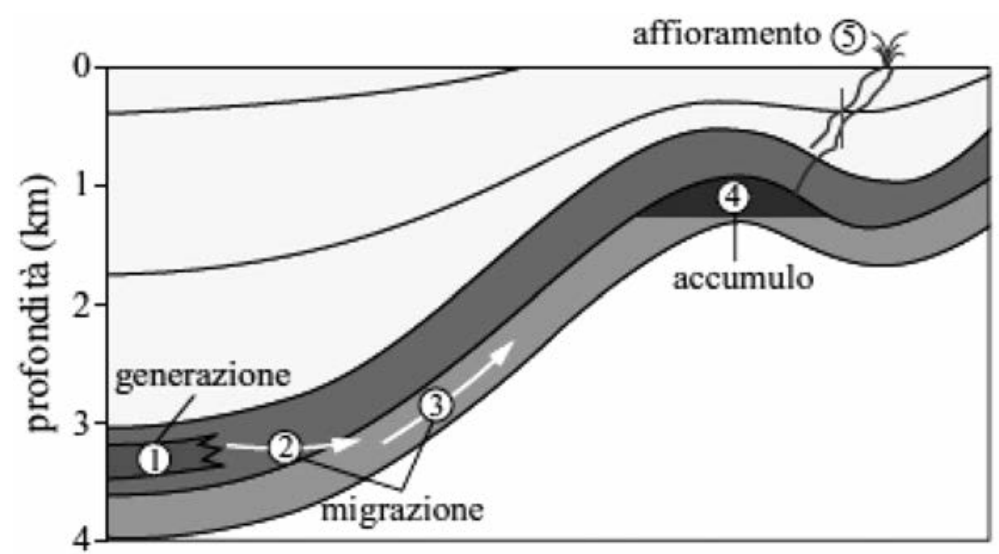

Fig. 3 - Schema semplificato dei rapporti laterali tra area di generazione degli idrocarburi (1), migrazione laterale (2 e 3) ed accumulo. Occasionalmente, gli idrocarburi possono trovarsi in superficie, come manifestazioni (5).

(AA.VV., 2005, modificato).

L'olio, essendo più leggero, migra o lateralmente o verso l'alto all'interno delle vicine rocce permeabili e secondo le ipotesi più accreditate può avvenire o per spostamento di goccioline di solo olio o di olio ed acqua.

La migrazione può avvenire in tre diversi modi:

- migrazione primaria: all'interno della roccia madre attraverso micro e macrofratture;

- migrazione secondaria: consiste nello spostamento degli idrocarburi nella roccia serbatoio attraverso i pori delle rocce permeabili adiacenti;

- dismigrazione: l'ultimo tipo di migrazione avviene nel caso in cui le 
rocce serbatoio e quelle che costituiscono la copertura (seal), vengano profondamente deformate da eventi tettonici per cui gli idrocarburi migrano ancora una volta fino a trovare nuovi reservoir.

In ogni caso, nel processo di migrazione un ruolo significativo viene svolto dal gas, che favorisce il trasporto dell'olio verso la roccia serbatoio.

Un aspetto di particolare importanza riguarda la distanza di migrazione, come per esempio nel caso del giacimento di Danish North Sea Basin (Mare del Nord), dove gli idrocarburi per migrare dall'area di generazione a quella di accumulo, hanno percorso circa $74 \mathrm{~km}$.

\section{RESERVOIR}

Gli idrocarburi, subito dopo la generazione, iniziano il processo di migrazione fino a quando non trovano una roccia "serbatoio" in cui si possono accumulare (Fig. 4).

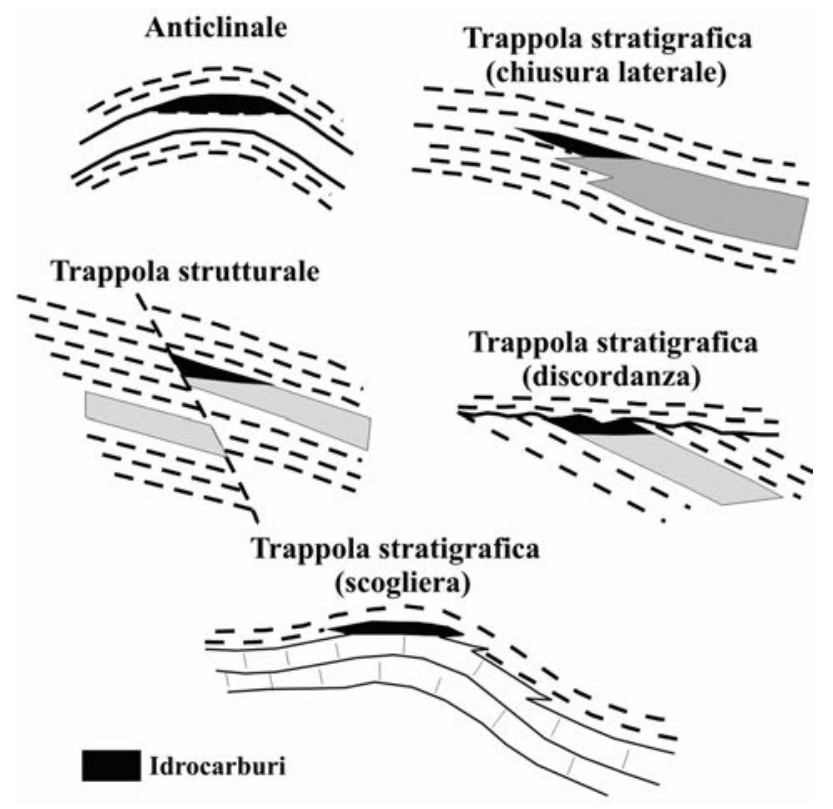

Fig. 4 - Esempi di tipi di trappole, dove si possono accumulare gli idrocarburi. 
Le caratteristiche che deve avere un reservoir (petrofisica), perché possa accumulare idrocarburi, sono strettamente legate alla porosità (percentuale dei pori presenti) ed alla permeabilità (capacità della roccia di farsi attraversare da un fluido), quest'ultima influenzata dalle interconnessioni dei pori e delle fratture.

Quasi la totalità dei reservoir sono di origine sedimentaria e quindi di natura detritica (clastica) o costituita da carbonato di calcio (rocce carbonatiche).

Le rocce di origine clastica possono essere incoerenti (es. sabbie) o coerenti (es. arenarie) e provengono ambedue dallo smantellamento di rocce preesistenti.

La granulometria può variare da $0,1 \mathrm{~mm}$ (es. argille) ad oltre 2 $\mathrm{mm}$ (es. sabbie) ed appare evidente come la porosità della roccia sia fortemente condizionata dalle dimensioni e dalla eterogeneità dei granuli.

Le rocce carbonatiche si generano in diversi ambienti; molto importanti ai fini degli accumuli di idrocarburi sono quelle che derivano dall'azione di organismi costruttori che danno origine a grandi barriere coralline o a vere e proprie scogliere, rappresentando dei reservoir ideali, caratterizzati da porosità e permeabilità particolarmente elevate.

In casi particolarmente rari, gli idrocarburi sono stati rinvenuti anche in altri tipi di rocce (magmatiche o metamorfiche) ma a causa delle caratteristiche petrofisiche di queste rocce (bassa porosità e permeabilità) non hanno mai avuto un'importanza economica significativa.

Le zone in cui gli idrocarburi si accumulano vengono definite "trappole" e possono essere classificate in strutturali o stratigrafiche.

Le trappole strutturali sono caratterizzate da una particolare conformazione geometrica della roccia nel sottosuolo (Fig. 4).

I reservoir infatti, devono avere una forma concava verso il basso ed essere limitati superiormente da una roccia a grana fine di natura argillosa o gessosa, che per le sue caratteristiche petrofisiche costituisca un livello impermeabile al passaggio di ogni tipo di fluido (seal).

Il limite inferiore della trappola può essere dato da un livello impermeabile, ma più spesso è caratterizzato dalla presenza di acqua salata (acquifero), secondo una normale stratificazione che dall'alto in basso prevede: gas, condensati, petrolio, acqua.

Il passaggio idrocarburi/acqua è quasi sempre orizzontale è viene definito come tavola acqua/gas o tavola acqua/olio. 
Le trappole stratigrafiche (Fig. 4) vengono suddivise in primarie, contemporanee al sedimento e secondarie, originatesi dopo la sedimentazione.

Tra gli esempi più significativi del primo gruppo, ricordiamo le scogliere carbonatiche e i canali fluviali e deltizi.

Nel primo caso la roccia biocostruita ha in genere un'alta porosità ed una elevata permeabilità. Spesso, associati a questi reservoir, è possibile riconoscere anche la presenza delle rocce madri (source rock).

Nel secondo caso, invece, si tratta di più o meno spesse sequenze clastiche deposte all'interno di incisioni sottomarine, provocate da forti correnti fluviali erosive.

Le correnti successive, più deboli, o i cambi di energia delle correnti erosive suddette favoriscono la deposizione all'interno degli stessi canali (es. Delta del Nilo - Egitto) o al loro sbocco sulle piane marine abissali.

Le trappole stratigrafiche secondarie si formano in coincidenza di discordanze (Fig. 4) a seguito di eventi tettonici (sollevamenti seguiti da erosioni o mancate deposizioni), successivamente ricoperte da sedimenti più recenti. Un esempio di questo tipo di trappole sono i giacimenti ad olio dell'Algeria Orientale, ubicati in coincidenza della discordanza ercinica, all'interno dei depositi del Trias superiore (es. Illizi basin).

\section{ROCCE DI COPERTURA}

Le rocce di copertura o "seal rock", sono costituite da rocce di origine sedimentaria a grana molto fine, in genere di tipo argillitica, che hanno la proprietà di impedire il passaggio dei fluidi, costituendo una vera e propria barriera invalicabile.

Dal punto di vista della ricerca petrolifera le rocce di copertura devono essere caratterizzate da una permeabilità trascurabile, molto bassa, non superiore a $10^{-4}$ darcy.

Oltre alla bassissima permeabilità, al di sotto delle seal rock non deve essere presente un gradiente di pressione particolarmente alto, per non compromettere la tenuta stessa della copertura, inoltre devono avere una elevata plasticità.

Appare evidente che anche lo spessore delle rocce di copertura influenza in modo significativo la tenuta. 
Non vi sono regole precise, tanto che in caso di provincie petrolifere poco deformate è sufficiente un "seal" di pochi metri (es. Arabia Saudita) mentre in altre aree si possono raggiungere spessori dell'ordine di diverse centinaia di metri (es. Romania).

È evidente che la migliore efficienza delle rocce di copertura viene raggiunta nel caso in cui abbiano una grande estensione laterale, una composizione litologica molto omogenea ed una plasticità elevata.

Le migliori rocce che rispondono a queste caratteristiche petrofisiche sono le argilliti e le evaporiti che, anche se sottoposte ad elevate sollecitazioni tettoniche, tendono a deformarsi plasticamente senza mai fratturarsi.

\section{LE ANALISI DEL POTENZIALE MINERARIO E DEL RISCHIO ASSOCIATO}

Malgrado i diversi tipi di studio che possono essere condotti in una determinata area, la probabilità di scoperta è sempre molta bassa (circa il 10\%) ed è quindi inevitabile che prima di perforare un pozzo si cerchi di tenere conto del rischio associato.

Per questo motivo è prassi comune utilizzare modelli statisticoprobabilistici per definire la percentuale di successo in una determinata area.

I parametri utilizzati tengono naturalmente conto della presenza della roccia madre, della porosità, della permeabilità, dalla presenza di una trappola e di una copertura impermeabile e soprattutto delle caratteristiche petrofisiche della roccia serbatoio. Se manca uno solo di questi elementi, la probabilità di fare una scoperta è praticamente nulla. La realtà spesso è molto complessa, in quanto entra in gioco anche l'efficienza di ogni singolo elemento.

\section{LA VALUTAZIONE DEI GIACIMENTI E IL CALCOLO DELLE RISERVE}

Nel momento in cui viene scoperto un nuovo giacimento di idrocarburi, devono essere eseguiti degli studi in modo da definire la dimensione, il tipo di idrocarburo contenuto (gas od olio), la temperatura, la pressione, la porosità e la permeabilità nella roccia che contiene gli idrocarburi ed anche delle rocce circostanti. 
Vengono anche condotti alcuni test che permettono di valutare la capacità produttiva dei reservoir al fine di pianificare le modalità e i tempi della produzione.

Un discorso a parte merita il calcolo delle riserve di un potenziale giacimento, in quanto una scoperta di idrocarburi è veramente tale nella misura in cui esistano le condizioni economiche per una messa in produzione e quindi per lo sviluppo.

Solo gli accumuli economicamente producibili possono essere considerati "riserve".

Per valutare i volumi di idrocarburi producibili in una determinata area bisogna calcolare:

- il volume di roccia che contiene gli idrocarburi (Rock Volume);

- il Net Pay che rappresenta lo spessore mineralizzato;

- la porosità;

- la saturazione in acqua $(\mathrm{Sw})$;

- il fattore di recupero (RF).

Il limite di questo tipo di valutazione è che spesso i valori di alcuni parametri sono il risultato di una valutazione regionale o statistica, e non del singolo prospect in esame.

Spesso, infatti, nel caso della porosità, della saturazione in acqua o della dimensione del giacimento, si utilizzano approcci probabilistici, caratterizzati da un valore minimo, medio e massimo.

L'approccio esplorativo al calcolo dei volumi è quindi di tipo statistico, i parametri suddetti e il tasso di variabilità (min, med, max) vengono inseriti in alcuni software di calcolo dedicati (es. REP - LogCom E\&P Ltd), che forniscono una stima degli idrocarburi in posto secondo una curva di distribuzione.

Quando si valuta una determinata area, riveste un ruolo particolarmente significativo capire qual è la percentuale di idrocarburi recuperabili. A tal proposito la geologia e l'ingegneria del petrolio distinguono le riserve in accertate (proved), probabili (probable) e possibili (possible):

1. le riserve accertate sono quelle per le quali si ha una certezza del recupero;

2. le riserve probabili sono quelle che si suppone possano essere recuperate anche se ancora mancano degli studi di giacimento che lo confermino;

3. le riserve possibili sono quelle incerte dal punto di vista tecnico.

Un'ulteriore complessità è data dalla modalità del recupero degli idrocarburi, in quanto può avvenire per: 
1. recupero primario, utilizzando la pressione esistente nel giacimento; il tasso di recupero primario si aggira in media sul $20 \%$;

2. recupero secondario, mediante l'estrazione assistita con pressurizzazione artificiale del giacimento, e con l'iniezione di gas inerti o di acqua; il tasso di recupero arriva al $30-35 \%$;

3. recupero terziario, mediante l'estrazione assistita sia con pressurizzazione artificiale del giacimento, sia con riscaldamento del greggio in situ, mediante l'iniezione di vapor d'acqua, oppure utilizzando prodotti chimici fluidificanti; il tasso di recupero giunge in tal caso al $40-45 \%$.

Nel caso degli idrocarburi gassosi contenuti in un reservoir sabbioso, la percentuale recuperabile è in media del 70\% circa anche se in qualche caso si può raggiungere l' $80-90 \%$.

Una volta calcolati i volumi e le riserve, rimane da valutare il potenziale giacimento anche dal punto di vista economico, per esprimere la redditività dell'investimento necessario per sviluppare l'accumulo.

Il risultato finale di quest'analisi è il cosiddetto EMV (Expected Mentary Value) che tiene conto delle variabili geologiche, tecniche ed economiche del progetto.

\section{IDROCARBURI NON CONVENZIONALI}

Nel corso degli ultimi decenni, grazie al notevole progresso della tecnologia, la ricerca petrolifera si è indirizzata anche verso quegli idrocarburi definiti non convenzionali.

Secondo recenti stime si ipotizza che le riserve di idrocarburi liquidi sono pari a circa 1.200 miliardi di barili; per quelli gassosi le riserve sarebbero pari a circa 1.224 miliardi di metri cubi.

Gli idrocarburi non convenzionali sono oggi rappresentati dagli oli pesanti, dalle oil sands e dalle gas shale.

L'olio pesante è caratterizzato da un alto contenuto in zolfo e azoto e da una bassa mobilità, si riviene in genere in depositi sabbiosi e a profondità modesta.

Il giacimento più rappresentativo è quello di Faja del Orinico in Venezuela che stime recenti hanno valutato avere riserve pari a 1.200 miliardi di barili, pari a quelle di olio convenzionale oggi certificate in tutto il mondo. 
Questi idrocarburi non convenzionali sono immersi in sabbie con una porosità media del $30 \%$ poste ad una profondità di circa $600 \mathrm{~m}$.

Le Oil Sands sono sabbie incoerenti e a bassa porosità che contengono olio, uno degli esempi più significativi si trova in Canada, nello stato dell'Alberta, nel giacimento Athabasca che è il più grande al mondo con queste caratteristiche. È costituito da livelli di sabbia spessa circa $60 \mathrm{~m}$, con una profondità variabile da $0 \mathrm{~m}$ a $600 \mathrm{~m}$ e si estende su un'area di circa $144.000 \mathrm{~km}^{2}$ con riserve pari a circa 1.7 trillioni di barili.

Le shale gas sono delle rocce scistose a grana molto fine estremamente compatte (argilliti) che contengono idrocarburi gassosi in grande quantità. Per potere produrre questo gas è necessario procedere con la fratturazione della roccia, mediante l'immissione di acqua ad alta pressione.

Le shale gas insieme al "tight gas" (sabbie compatte) e al "coalbed methane" (carbone) rappresentano ormai un'opportunità per molti paesi; ad esempio lo sviluppo e la produzione di questo tipo di gas negli Stati Uniti rappresenta circa il $60 \%$ delle riserve onshore tecnicamente recuperabili, secondo le stime del Dipartimento dell'Energia.

I giacimenti più importanti di shale gas negli Stati Uniti sono il Barnett in Texas, l'Haynesville in Luosiana e Texas, il Fayetteville in Arkansas e il Marcellus in Pennsylvania e in alcuni stati limitrofi.

In particolare, il Marcellus viene considerato il più importante, tra i principali player con riserve pari a circa 14.800 miliardi di metri cubi di gas.

Accanto agli oli tradizionali, nel corso delle ricerche petrolifere, sono sempre stati individuati giacimenti di oli pesanti e molto viscosi, che non venivano sempre messi in produzione a causa della mancanza di una tecnologica economicamente adeguata.

Nel corso degli ultimi decenni la percentuale di olio non convenzionale è aumentata in modo evidente, fino a raggiungere il 10\% circa della produzione mondiale. Questo consistente aumento è stato possibile grazie all'applicazione di nuove modalità di produzione, come ad esempio il Cycle Steam Stimulation (CSS), che consiste nell'immettere nei giacimenti grandi quantità di vapore in modo ciclico, con risultati significativi, grazie anche allo sviluppo della tecnologia per la perforazione di pozzi orizzontali.

Un'altra tecnologia applicata di recente è la Cold Heavy Oil 
Production with Sand (CHOPS) che consente di produrre olio a viscosità non molto alta senza l'utilizzo di fluidi ad alte temperature, od ancora con la metodologia Steam Assisted Gravity Drainage (SAGD) che consiste nel favorire un drenaggio dell'olio per gravità grazie all'uso del vapore.

\section{ACQUISIZIONE DI UN TITOLO MINERARIO E TIPOLOGIA DI CONTRATTI}

Per potere operare in una determinata area è necessario acquisire un "titolo minerario" il quale viene identificato geograficamente da un sistema di coordinate.

La titolarità può essere ottenuta in tre diversi modi ed in particolare per:

1. partecipazione ad una gara internazionale (bid round) in cui ogni compagnia presenta una proposta e lo stato seleziona quella che garantisce il miglior investimento;

2. accordo con un'altra compagnia petrolifera che detiene le operazioni in quel blocco (farm out);

3. trattativa diretta con uno Stato;

4. mediante la presentazione di un'apposita Istanza di Permesso (Italia).

Il primo tipo di possibilità, attraverso gara internazionale, è molto diffuso ma può presentare degli aspetti problematici legati alla frequenza, qualità, disponibilità di aree.

La politica del farm-out (cessione di una parte o della totalità di una quota di un titolo minerario) è legata alla necessità di alcune compagnie di ridurre la loro esposizione finanziaria verso un singolo permesso di ricerca.

L'opzione più economica è rappresentata dalla trattativa diretta con un governo, questo consente ad una compagnia di investigare una determinata area senza nessun tipo di obbligo e di spesa.

È evidente l'impegno di consegnare allo Stato copia di tutti i lavori effettuati in modo anche da opzionare eventuali aree di interesse.

L'acquisizione di un permesso esplorativo prevede, in genere, una vigenza articolata in tre fasi distinte.

Nel primo periodo, che ha una durata in genere di 4 o 5 anni, la 
compagnia ha l'obbligo del rispetto degli impegni presi, che normalmente consistono nell'acquisizione di sismica a riflessione $2 \mathrm{D}$ o $3 \mathrm{D}$ e di un pozzo esplorativo.

Il passaggio al periodo successivo comporta una riduzione dell'area originaria del Permesso di ricerca di una percentuale del $25-30 \%$ (Relinquishment).

I due periodi successivi non sono obbligatori ma sono legati ai risultati della prima fase e se una compagnia petrolifera decide di accedervi, deve assumersi altri obblighi che comportano ulteriori impegni finanziari.

In generale, durante il periodo di ricerca, le compagnie devono eseguire degli studi geologici e geofisici per verificare la presenza o meno di tutti quegli elementi che consentono di valutare il potenziale petrolifero dell'area in esame.

La perforazione di uno o più pozzi esplorativi permette di verificare o meno la presenza di un giacimento e quindi delle potenziali riserve presenti.

Nel caso di una scoperta si passa poi allo sviluppo del giacimento, con la perforazione di altri pozzi e con la realizzazione delle infrastrutture necessarie per la produzione e il trasferimento (pipeline) verso i siti di trattamento.

Appare quanto mai ovvio che i rapporti tra compagnia petrolifera e un Paese, in riferimento alle attività esplorative e, in caso di successo, di produzione, devono essere regolate da differenti tipi di contratti, ed in particolare:

Royalty e tasse (Concession Agreement): vengono versate allo Stato royalty in percentuale sulla quantità di idrocarburi prodotti (es 8$10 \%$ ) e pagate le tasse sul reddito;

Production Sharing Agreement: la Compagnia assume i rischi della ricerca ed i costi e gli investimenti di sviluppo vengono recuperati, in caso di produzione, per una certa percentuale (oil cost, es 40\%). La produzione rimanente viene divisa tra Compagnia e Società di Stato secondo percentuali variabili in funzione della produzione (profit split) (es 70/30 fino a 85/15). In questi tipi di contratti vengono normalmente riconosciuti bonus di produzione;

Contratti di buy-back: la Compagnia opera come Società di servizio ed in caso di successo viene remunerata per i costi e gli investimenti sostenuti con una quota di produzione fino ad ottenere una redditività predefinita. 


\section{I COSTI DELL'ESPLORAZIONE PETROLIFERA}

La valutazione dei costi per la sola fase esplorativa non è così semplice come possa sembrare, anche perché sono parametri estremamente variabili e dipendono dal paese in cui si opera, dall'ubicazione del permesso di ricerca in mare (offshore) o in terra (onshore), dalla topografia del terreno, dalla disponibilità dell'attrezzatura nell'aree limitrofe e dalla profondità dell'obiettivo da raggiungere.

L'impatto maggiore sui costi è dato dagli studi geofisici ed in particolare dall'acquisizione sismica e dalla perforazione dei pozzi esplorativi (Fig. 5).

Per quanto riguarda l'acquisizione sismica, il costo cambia in funzione non solo del tipo (2D o 3D), ma anche della conformazione topografica (collina o pianura) e dell'ambiente (marino o terrestre).

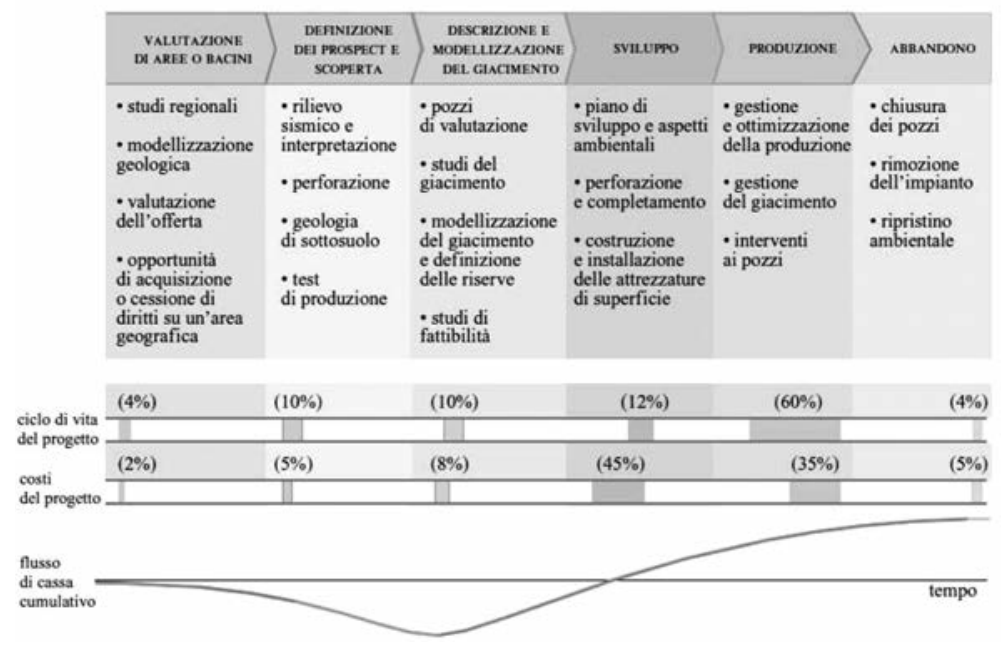

Fig. 5 - Schema semplificato delle diverse fasi della ricerca petrolifera. (Edison, 2001).

Per fare un esempio, in Italia l'acquisizione sismica 2D in mare ha un costo medio di circa $20.000 \$ / / \mathrm{km}$, all'estero (bacino Mediterraneo) di circa $25.000 \$ / / \mathrm{km}$. L'acquisizione di sismica 3D può costare anche $45.000 \$ \mathrm{kmq}$.

L'acquisizione in terra dipende naturalmente dalla topografia, in pianura il costo di acquisizione è di circa $7.000 \$ / / \mathrm{km}$, in un ambiente collinare o montuoso può raggiungere anche i 20.000 \$//km. 
È quanto meno ovvio che l'acquisizione 3D abbia costi di acquisizione molto più alti, arrivando anche a $15.000 \$ / / \mathrm{kmq}$ e 20.000 $\$ / / \mathrm{kmq}$ in terra.

Anche i costi relativi alla perforazione dei pozzi dipende da molti fattori, tra i quali l'ubicazione (onshore o offshore) e il Paese dove si opera.

La perforazione di un pozzo esplorativo nel deserto egiziano con un obiettivo a circa $3500 \mathrm{~m}$ di profondità costa circa 1,5 milioni di dollari, un pozzo in Algeria e con caratteristiche geologiche molto simili, può costare anche 15 milioni di dollari.

Se il pozzo è ubicato in mare (offshore), l'utilizzo di una piattaforma (semisub) costa mediamente $500.000 \$$ /giorno, mentre una nave attrezzata per la perforazione (drillship) arriva fino ad 1 milione $\$$ /giorno. Per quanto riguarda invece i jack-up i costi attuali sono dell'ordine dei $70.000 \$ /$ giorno.

\section{LE RISERVE DI OLIO}

Trattare questo argomento richiede necessariamente l'utilizzo di informazioni attendibili che tengano anche conto delle certificazioni internazionali che vengono rilasciate alle compagnie petrolifere, da apposite società internazionali di valutazione.

Uno degli annual report più diffusi ed utilizzati, è quello redatto dalla British Petroleum (BP) e pubblicato ogni anno (BP Statistical Review of World Energy, June 2011) e che si riferisce alle informazione relative al 2010.

Per quanto riguarda le riserve di petrolio alla fine del 2010, risultavano certificate circa 1.333,1 miliardi di barili, con un incremento negli ultimi 30 anni di circa il 32\% (Fig. 6).

In questo contesto è da rilevare che le riserve maggiori sono localizzare in Arabia Saudita (264,6 Miliardi di barili), in Iran 137,6, in Iraq 115 e in Kuwait 101. In Africa le riserve maggiori sono in Libia (44 miliardi di barili), in Nigeria $(37,2)$, in Angola $(13,5)$ e in Algeria $(12,2)$. Nel continente americano abbiamo il Venezuela con 172,3 miliardi di barili e il Brasile con 12,9. Per quanto concerne l'Italia, sono state accertate riserve per 1 miliardo di barili di petrolio.

I paesi produttori che hanno registrato una riduzione nelle riserve sono il Messico e gli USA. 
Distribution of proved reserves in 1990, 2000 and 2010 Pistributage

\section{E. Middie East}

- S. \& Cent. America

- Europe \& Eurasia

Africa

in North America
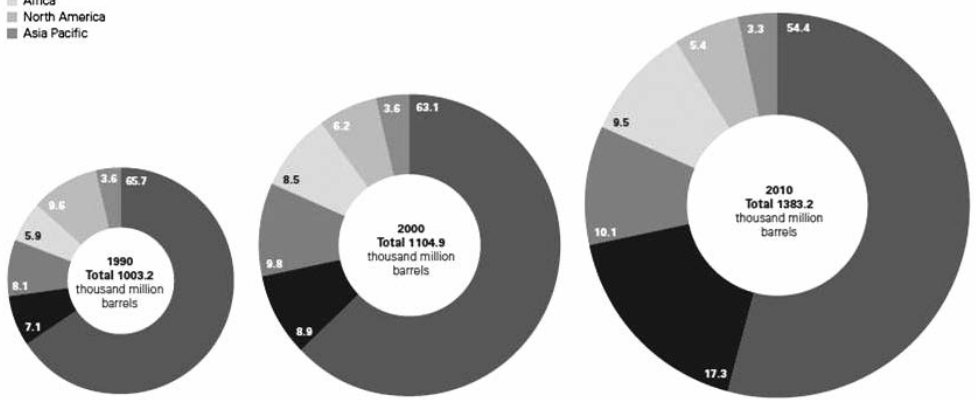

Fig. 6 - Distribuzione delle riserve dell'olio nel 1990, 2000 e 2010

(valore espresso in percentuale). (British Petroleum, 2011, modificato).

\section{LE RISERVE DI GAS}

Le riserve di Gas nel mondo ammontano nel 2010 a circa 187.490 miliardi di metri cubi con un incremento rispetto al 1989 di circa il $50 \%$ (Fig. 7).

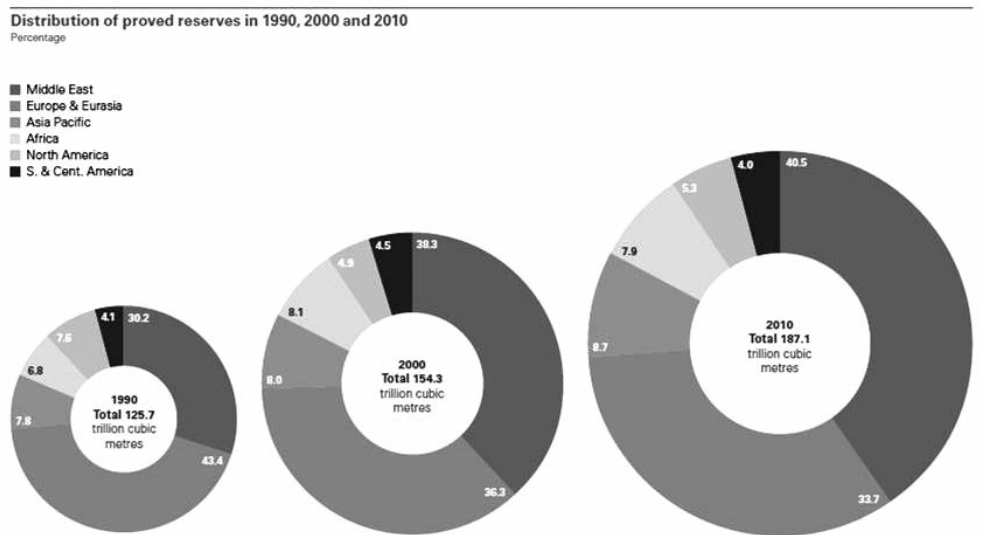

Fig. 7 - Distribuzione delle riserve del gas nel 1990, 2000 e 2010 (valore espresso in percentuale). (British Petroleum, 2011, modificato). 
La maggior parte delle riserve sono localizzate nel medio-oriente $(40,8 \%)$ e nell'Europa centro-orientale $(33,7 \%)$.

I paesi con le riserve più importanti sono rappresentati dalla Russia (44 trilioni metri cubi), l'Iran $(29,6)$ e il Qatar $(25,3)$.

\section{I CONSUMI}

La recessione economica ha influito sulla riduzione dei consumi di idrocarburi soprattutto nel 2009 con una percentuale di circa 1,1\%, che si è tradotta anche con una diminuizione di $\mathrm{CO}_{2}$ immessa nell'atmosfera, pari ai livelli del 1998. Nel 2010 invece si è registrata una lieve ripresa, soprattutto nell'estremo oriente (Fig. 8).

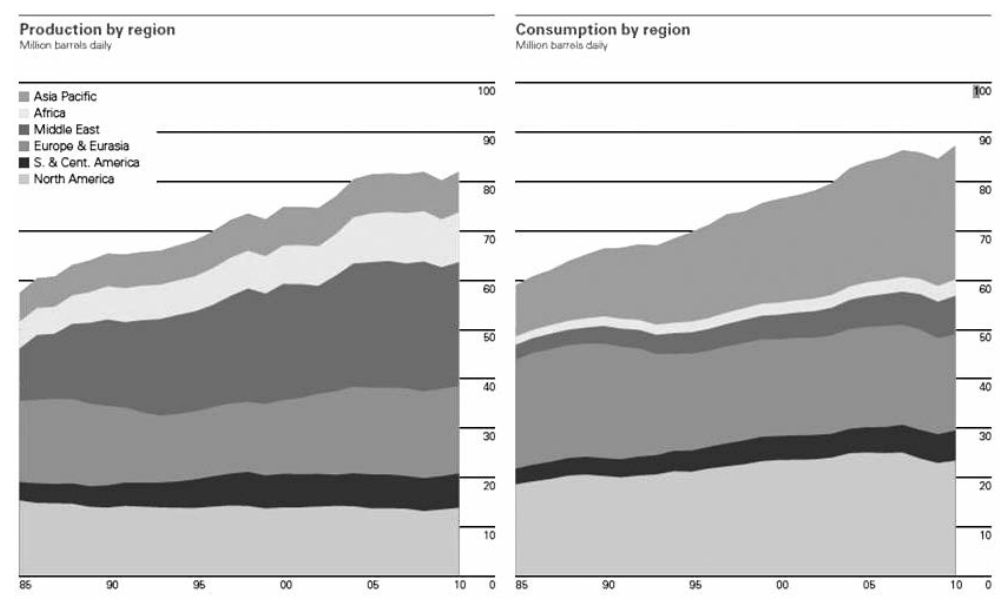

Fig. 8 - Il primo grafico indica la produzione/giorno di olio suddivisa per regione tra il 1986 e il 2010; il secondo grafico indica il consumo/giorno, sempre per lo stesso periodo di tempo. (British Petroleum, 2011, modificato).

Il consumo di olio negli ultimi 20 anni, è aumentato progressivamente da circa 60 milioni di barili/giorno del 1984, a circa 83 milioni barili/giorno del 2009, raggiungendo gli 87 milioni barili/giorno nel 2010.

La riduzione dei consumi nel 2009 pari a circa 1,2 milioni di barili $(-1,7 \%)$ è da riferire alla recente crisi economica, con un calo dei consumi in alcuni paesi anche del 10\% (Giappone, Irlanda e Bielorussia). A livello continentale, la riduzione dei consumi più marcata si è avuta 
in America Settentrionale (-4,7\%), in Europa $(-4,2 \%)$ e Medio Oriente $(-3,8 \%)$.

Con il 2010, la lieve ripresa economica ha comportato un aumento consistente dei consumi in Cina $(+10 \%)$, a Singapore $(+11 \%)$, in Vietnam $(+10 \%)$ e in Qatar $(+18 \%)$.

Per quanto riguarda il consumo di gas (Fig. 9) durante il 2009 sono stati utilizzati circa 2950,2 miliardi di metri cubi, con una riduzione dei consumi rispetto all'anno precedente di circa il 2,1\%. Questo calo si è registrato in tutti i continenti con percentuale diversa, ma in modo più marcato in Europa $(-6,8 \%)$, in Medio Oriente $(-4,4 \%)$, in America Meridionale (-4,2\%), nell'Asia del Pacifico (-3,4\%) e nel Nord America (-1,2\%).

Anche per il gas nel 2010 si registra un aumento dei consumi con circa 3169 miliardi di metri cubi, con un incremento percentuale rispetto al 2009 pari al $7,4 \%$.
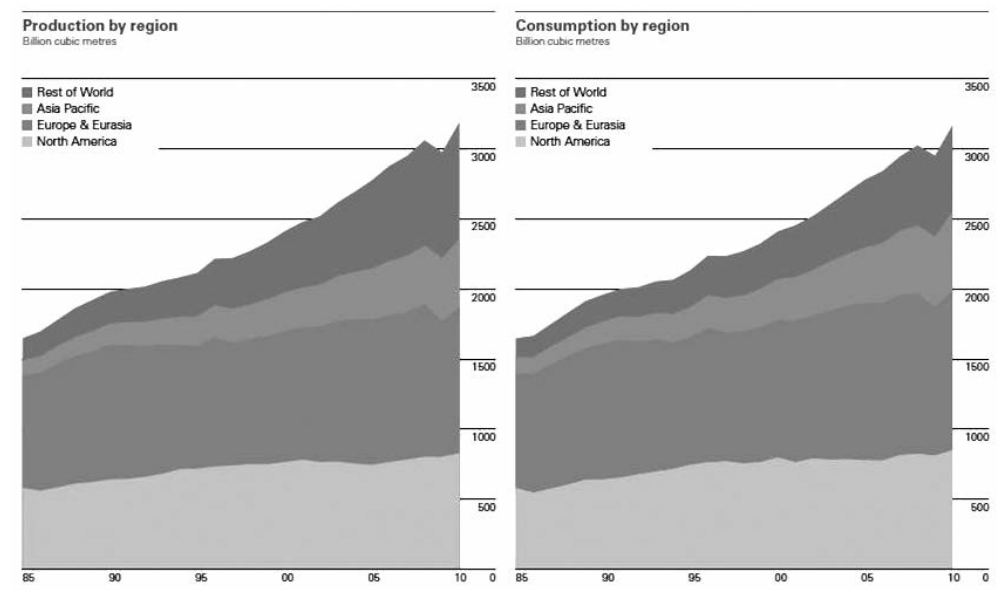

Fig. 9 - Il primo grafico indica la produzione/giorno di gas divisa per regione tra il 1986 e il 2010; il secondo grafico indica il consumo/giorno,

sempre per lo stesso periodo di tempo. (British Petroleum, 2011, modificato).

\section{L'EVOLUZIONE DEL PREZZO DEL PETROLIO}

La ricerca petrolifera fin dagli albori, è sempre stata interessata da un andamento ciclico e allo stesso modo, anche il petrolio è stato soggetto a forti oscillazioni del prezzo (Fig. 10). Nel secolo XIX con l'inizio della 
prima fase esplorativa (1861) il costo del petrolio era molto alto, pari a quasi $8 \$$ al barile. I valori sono rimasti relativamente alti fino al 1880 circa quando l'Unione Sovietica ha messo in produzione i primi campi. La prima vera e propria riduzione del prezzo del greggio coincide con la crisi economica del 1929, con il prezzo che raggiunge i $10 \$$ al barile.

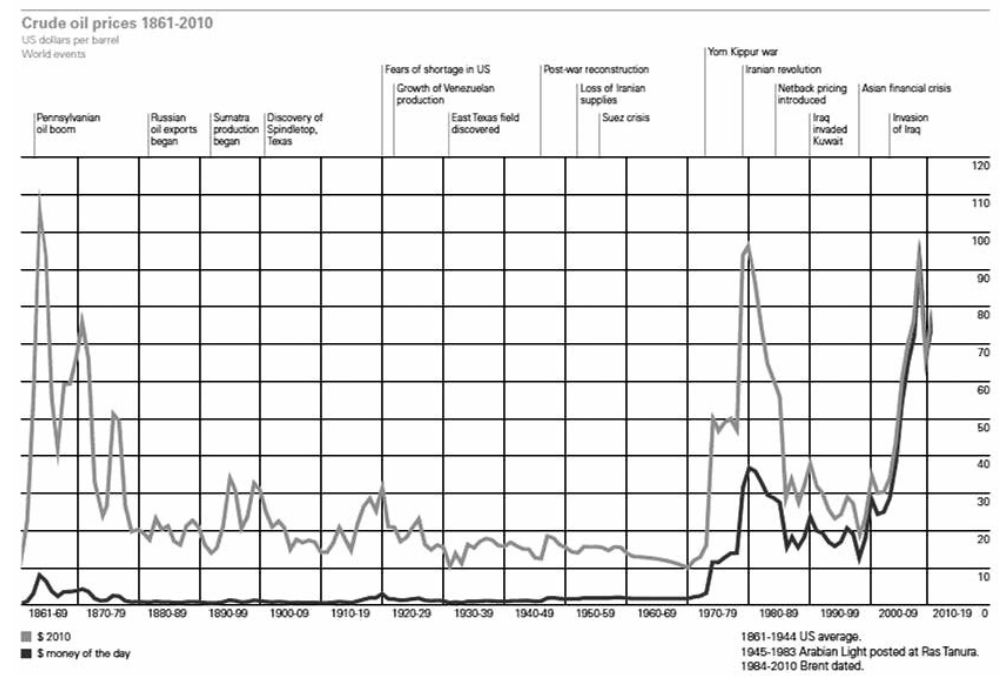

Fig. 10 - Il grafico indica il costo del petrolio tra il 1861 e il 2009.

È possibile osservare due curve, la curva chiara è riferita al costo attualizzato del barile, quella più scura al valore reale della vendita nell'anno in questione.

(British Petroleum, 2011, modificato).

Tra il 1930 e il 1969, il valore del barile oscilla sempre tra i $10 \$$ e i $20 \$$ e solo con la Guerra del Kippur e la Rivoluzione Iraniana, si registra un consistente aumento del prezzo, fino a raggiungere nel 1980, i $38 \$$ al barile.

Successivamente a questi eventi, la prima invasione del Kuwait e la crisi asiatica del 2000, hanno prodotto un'ulteriore oscillazione, fino a toccare nel 1999, i $35 \$$ al barile.

La seconda invasione dell'Iraq ha prodotto un ulteriore aumento del costo del petrolio, fino a raggiungere il prezzo di oltre $90 \$$. Con l'inizio del 2009 il prezzo del barile era pari a $40 \$$ per poi raggiungere $i$ $70 \$$ nella metà di novembre dello stesso anno. Con il 2010, il prezzo si è in qualche modo stabilizzato sui $115-120 \$$ al barile. 


\section{GLI IDROCARBURI E GLI SCENARI FUTURI}

Quanto dureranno ancora gli idrocarburi nessuno può affermarlo con certezza. Possiamo certamente ammettere che le riserve accertate e certificate al 2010 sono pari a 1.383 miliardi di barili per il petrolio. Il consumo giornaliero nel mondo è pari a circa 87 milioni di barili, pari a circa 32 miliardi di barili in un anno.

Per quanto riguarda il gas, le riserve accertate sono pari a 187 trilioni di metri cubi e nel 2010 sono stati consumati circa 3170 miliardi di metri cubi.

Se non vi fossero ulteriori scoperte e tenendo conto delle riserve e dei consumi si avrebbero idrocarburi sufficienti per circa 60 anni. È tuttavia importante rilevare che in queste valutazioni non rientrano gli idrocarburi non convenzionali, che non vengono ancora adeguatamente sfruttati sia per gli alti costi che per il forte impatto ambientale.

Per fare alcuni esempi, nel giacimento oil sands di Athabasca (Canada) sono stati stimate riserve pari a circa 178 miliardi di barili, estraibili senza l'ausilio di tecnologie speciali od ancora il giacimento oil sands di Orinoco (Venezuela), dove sono stati stimate riserve recuperabili pari a 513 miliardi di barili.

La stessa valutazione deve essere fatta anche per gli idrocarburi gassosi non convenzionali, dove sulla base di una stima approssimativa, si ipotizzano riserve potenziali pari a 180.000 miliardi di metri cubi.

Rimane certamente l'incertezza legata alle riserve reali certificate e all'autonomia che l'intero sistema produttivo mondiale può avere fino all'esaurimento completo degli idrocarburi.

A tal proposito, nel 1956, un geofisico americano della Shell che lavorava in Texas (Marion King Hubbert), ha cercato di valutare la durata degli idrocarburi tenendo conto della produzione annuale e delle riserve di idrocarburi sia convenzionali che non, in modo da prevedere quando si sarebbe giunti al picco di produzione, oltre il quale gli idrocarburi vanno in esaurimento. Sulla base di questa ipotesi (curva di Hubbert), il picco è stato raggiunto proprio in questi anni, per cui le prossime scoperte non saranno più sufficienti a rimpiazzare gli idrocarburi consumati. 


\section{BIBLIOGRAFIA DI RIFERIMENTO}

AA.VV. Enciclopedia degli Idrocarburi, Istituto dell'Enciclopedia Italiana Treccani, (2005).

P.A. Allen \& J.R. Allen, Basin analysis. Principles and applications. Oxford, Blackwell, (1990).

D.R. Baker, Organic geochemistry of Cherokee Group in southeastern Kansas and northeastern Oklahoma, American Association of Petroleum Geologists. Bulletin», 46 (1962), 1621-1642.

R.A. Baker, H.M. Gehman, W.R. James, D.A. Whit, Geological field number and size assessments of oil and gas plays, American Association of Petroleum Geologists. Bulletin, 68 (1984), 426-437.

A.W. Bally, R. Catalano, J. Oldow, Elementi di tettonica regionale. Evoluzione dei bacini sedimentari e delle catene montuose, Bologna, Pitagora (1985).

Z. Berger, Satellite bydrocarbon exploration. Interpretation and integration techniques, Berlin-New York, Springer (1994).

T. Bernhardsen, Geographic Information Systems. An introduction, Chichester, John Wiley (2002).

K.K. Bissada, L.W. Elrod, C.R. Robison, L.M. Darnell, H.M. Szymczyk and J.L. Trostle, Geochemical inversion. A modern approach to inferring source-rock identity from characteristics of accumulated oil and gas, Energy Exploration and Exploitation, 11 (1993), 295-328.

P. Blanc, J.Connan, Crude oils in reservoirs. The factors influencing their composition, in: Bordenave M.L. (edited by) Applied petroleum geochemistry, Paris, Technip (1993), 149-174.

J.M. Bourdaire, R.J. Byramjee \& R. Pattinson, Reserve assessment under uncertainty. A new approach, Oil \& Gas Journal, June, 135-140 (1985).

M.L. Bordenave, J. Espitaliè, P. Leplate and J.L. Oudin, Screening techniques for source rock evaluation, in: Bordenave M.L. (edited by) Applied petroleum geochemistry, Paris, Technip (1993), 217-278.

British Petroleum, Statistical review of world energy: full report. What's inside? (2011).

L. Cagniard, Basic theory of the magnetotelluric method of geophysical prospecting, Geophysics, 18 (1953), 605-635.

J.D. Campbell, Analysis and Management of Petroleum Investments: Risk, Taxes, and Time, $2^{\text {nd }}$ Ed., CPS Publishing, Norman, OK (1991).

J. Connan, Molecular geochemistry in oil exploration, in: Bordenave M.L. (edited by) Applied petroleum geochemistry, Paris, Technip (1993), 175-206.

J.P. Castagna, M.M. Backus (editors), Offset-dependent reflectivity. Theory and practice of AVO analysis, Tulsa (OK), Society of Exploration Geophysicists (1990). 
J.L. Clayton, P.J. Swetland Subaerial weathering of sedimentary organic matter, Geochimica et Cosmochimica Acta, 42 (1978), 305-312.

J. Connan, A.M. Cassou, Properties of gases and petroleum liquids derived from terrestrial kerogen at various maturation levels, Geochimica et Cosmochimica Acta, 44 (1980), 1-23.

E.C. Capen, The difficulty of assessing uncertainty, Journal of Petroleum Technology, 28 (1976), 843-850.

E.C. Capen, Dealing with exploration uncertainties, in: Steinmetz R. (edited by) The business of petroleum exploration, Tulsa (OK), American Association of Petroleum Geologists, 29-61 (1992).

F.E. Cotton, Investment indicators for profitability analysis must reflect recent economic development, Gas Journal», October (1983).

G.J. Demaison, G.T. Moore, Anoxic environments and oil source bed genesis, American Association of Petroleum, Geologists. Bulletin», 64 (1980), 1179-1209.

W.G. Dow, D.I. O'Connor, Kerogen maturity and type by reflected light microscopy applied to petroleum exploration, in: Staplin F.L. et al., How to assess maturation and paleotemperatures, Tulsa (OK), Society of Economic Paleontologists and Mineralogists (1982), 133-157.

S.A. Drury, Image interpretation in geology, London, Allen \& Unwin (1987).

A.E. Dunstan, B.T. Brooks, The science of petroleum. A comprehensive treatise of the principles and practice of the production, refining, transport and distribution of mineral oil, London, Oxford University Press (1938).

Eni, Enciclopedia del petrolio e del gas naturale, Roma, Colombo, 8v (2007).

W.A. England, A.S. Mackenzie, D.M. Mann, T.M. Quigley, The movement and entrapment of petroleum fluids in the subsurface, Journal of the Geological Society, 144 (1987), 327-347.

D.J. Forman, A.L. Hinde, Improved statistical method for assessment of undiscovered petroleum resources, American Association of Petroleum Geologists. Bulletin, 69 (1985), 106-118.

D.J. Forman, A.L. Hinde, Examination of the creaming methods of assessment applied to the Gippsland basin, offshore Australia, in: Rice D.D. (edited by) Oil and gas assessment. Methods and applications, Tulsa (OK), American Association of Petroleum Geologists, 101-110 (1986).

N.H. Foster, E.A. Beaumont (edited by), Remote sensing, Tulsa (OK), American Association of Petroleum Geologists (1992).

T.C. Frick, R.W. Taylor, Petroleum production handbook, New York, McGraw-Hill, 2v (1962).

R.A. Gallun, J.W. Stevenson, Fundamentals of oil and gas accounting, Tulsa (OK), PennWell (1986).

J.B. Gustavson, D.J. Murphy, Risk analysis in hydrocarbon appraisal, in: Proceedings of the Society of Petroleum Engineers hydrocarbon economics and evaluation (1989). 
B. Horsfield Practical criteria for classifying kerogens. Some observations from pyrolysis-gas chromatography, Geochimica et Cosmochimica Acta, 53 (1990), 891-901.

J.M. Hunt, Petroleum geochemistry and geology, New York, W.H. Freeman (1996).

R.W. Jones, Comparison of carbonate and shale source rocks, in: J. Palacas (edited by) Petroleum geochemistry and source rock potential of carbonate rocks, Tulsa (OK), American Association of Petroleum Geologists (1984), 163-180.

S.D. Killops, V.J. Killops, An introduction to organic geochemistry, New York, John Wiley (1993).

H.D. Klemme, Field size distribution related to basin characteristics, Oil \& Gas Journal, December, 168-176 (1983).

H.D. Klemme, Giant oil fields related to their geological setting. A possible guide to exploration, Bulletin of Canadian Petroleum Geology, 23 (1975), 30-66.

C.R. Landis, J.R. Castaño, Maturation and bulk chemical properties of a suite of solid bydrocarbons, Organic Geochemistry, 22 (1995), 137-149.

A.I. Levorsen, Geology of petroleum, San Francisco (CA) - London, W.H. Freeman (1967)

D. Leythaeuser, H.S. Poelchau, Expulsion of petroleum from Type III kerogen source rocks in gaseous solution. Modelling of solubility fractionation, in: England W.A., Fleet A.J. (edited by) Petroleum migration, London, Geological Society Special Publication, 59 (1991), 33-46.

L.B. Magoon, W.G. Dow, The petroleum system, in: Magoon L.B., Dow W.G. (edited by) The petroleum system. From source to trap, Tulsa (OK), American Association of Petroleum Geologists (1994), 3-24.

F.D. Mango The origin of light hydrocarbons in petroleum. A kinetic test of the steadystate catalytic bypothesis, Geochimica et Cosmochimica Acta, 54 (1990), 1315. 1323.

C.D. Masters, E.D. Attanasi, and D.H. Root, World petroleum assessment $\mathcal{E}$ analysis, in: Proceedings of the 14th World petroleum congress, Stavanger (Norway), Chichester-New York, John Wiley, 529-541 (1994).

L. Mattavelli, T. Ricchiuto, D. Grignani, M. Schoell, Geochemistry and habitat of natural gases in Po basin, Northern Italy, American Association of Petroleum Geologists. Bulletin, 67 (1983), 2239-2254.

D. Maune (edited by), Digital elevation model technologies and applications, Bethesda (MD), American Society for Photogrammetry and Remote Sensing (2001).

A.S. McKenzie, T.M. Quigley Principles of geochemical prospect appraisal, American Association of Petroleum Geologists. Bulletin, 72 (1988), 300-415.

R.E. Megill, An introduction to risk analysis, Tulsa (OK), PennWell (1984).

R.E. Megill, History of exploration expenditure in the United States, in: Steinmetz R. (edited by) The business of petroleum exploration, Tulsa (OK), American Association of Petroleum Geologists, 13-20 (1992).

K.E. Peters, J.M. Moldowan The biomarker guide. Interpreting molecular fossils in 
petroleum and ancient sediments, Englewood Cliffs (NJ), Prentice Hall (1993).

F.J. Pettijohn, Sedimentary rocks, New York, Harper \& Brothers (1957).

P.R. Rose, Dealing with risk and uncertainty in exploration. How can we improve?, American Association of Petroleum Geologists. Bulletin», 71 (1987), 1-16.

Royal Dutch Shell Group of Companies, The petroleum handbook, AmsterdamOxford-New York-Tokyo, Elsevier, (1987).

M. Schoell, Genetic characterization of natural gases, American Association of Petroleum Geologists. Bulletin, 67 (1983), 2225-2238.

W.K. Seifert, J.M. Moldowan Use of biological markers in petroleum exploration, in: Johns R.B. (edited by) Methods in geochemistry and geophysics, AmsterdamOxford, Elsevier, v. XXIV (1986), 261-290.

W.K. Seifert, J.M. Moldowan, Applications of steranes, terpanes, and monoaromatics to the maturation, migration, and source of crude oils, Geochimica et Cosmochimica Acta, 42 (1978), 77-95.

R.C. Selley, Elements of petroleum geology, San Diego (CA), Academic Press (1998).

R.E. Sheriff, Encyclopedic dictionary of applied geophysics, Tulsa (OK), Society of Exploration Geophysicists (2002).

D. Sluijk, M.H. Nederlof, Worldwide geological experience as a systematic basis for prospect appraisal, in: Demaison G., Murris R.J. (edited by) Petroleum geochemistry and basin evaluation, Tulsa $(\mathrm{OK})$, American Association of Petroleum Geologists, 15-26 (1984), symposium, Dallas (TX), 9-10 March, SPE.

B.P. Tissot, D.H. Welte, Petroleum formation and occurrence, Berlin, Springer (1984).

K.F.M. Thompson, Classification and thermal history of petroleum based on light bydrocarbons, Geochimica et Cosmochimica Acta, 47 (1983), 303-316.

D.W. Van Krevelen Coal. Typology, chemistry, physics, constitution, Amsterdam, Elsevier (1961).

D.W. Waples, The kinetics of in-reservoir oil destruction and gas formation. Constraints from experimental and empirical data, and from thermodynamics, Organic Geochemistry, 31 (2000), 553-575.

D.W. Waples, T. Machihara Biomarkers for geologists. A practical guide to the application of steranes and triterpanes in petroleum geology, Tulsa (OK), American Association of Petroleum Geologists (1991).

D.H. Welte, B. Horsfield, D.R. Baker (editors) Petroleum and basin evolution, Berlin, Springer (1997).

P.R. Wolf, B.A. Devitt, Elements of photogrammetry with applications in GIS, Boston (MA), McGraw-Hill (2000).

R.D. Woods, Long-term trends in oil and gas discovery rates in lower 48 states, American Association of Petroleum Geologists. Bulletin, 69 (1985), 1 321-1326. 